\title{
The Function Role of miR-181a in Chemosensitivity to Adriamycin by Targeting Bcl-2 in Low-Invasive Breast Cancer Cells
}

\author{
Ying Zhua,b Jianzhong Wu ${ }^{b}$ Shuchun Lic Rong Ma ${ }^{b}$ Haixia Cao ${ }^{b}$ Minghua Jid \\ Changwen Jing ${ }^{b}$ Jinhai Tang ${ }^{a}$ \\ aDepartment of General Surgery, the Affiliated Jiangsu Cancer Hospital, Nanjing Medical University, \\ ${ }^{\mathrm{b}}$ Research Center of Clinical Oncology, the Affiliated Jiangsu Cancer Hospital, Nanjing Medical \\ University, 'School of Pharmaceutical science, Nanjing University of Technology, dDepartment of \\ Radiotherapy, the Affiliated Jiangsu Cancer Hospital, Nanjing Medical University, Nanjing, Jiangsu, \\ P.R. China
}

\section{Key Words}

$\mathrm{miR}-181 \mathrm{a} \cdot$ Breast cancer $\bullet$ Chemosensitivity $\cdot$ Adriamycin $\bullet$ Bcl-2

\begin{abstract}
Objectives: miR-181a is involved in immunity, metabolism, tumor suppression or carcinogenesis reported by many other studies. However, its role in the development of chemosensitivity to adriamycin in low-invasive breast cancer cells remains unclear. The aim of this study is to define the function role of miR-181a in promoting the efficacy of adriamycin-based neoadjuvant chemotherapy. Methods: Cell survival analysis was detected by Cell Counting Kit- 8 assay. Apoptotic cells were quantitatively detected using FITC Annexin V apoptosis Detection Kit I. $\mathrm{BCl}-2$ protein expression was measured by western blot. Luciferase reporter vector with the putative BCL-2 3' untranslated region (3'UTR) was constructed to explore whether BCL-2 was a direct target gene of miR-181a. Real-time PCR was performed to test the expression of miR$181 \mathrm{a}$ and $\mathrm{BCl}-2$ in the selected breast cancer tissue samples. Results: The down-regulation of miR-181a decreased adriamycin-induced apoptosis in MCF-7 cells. Transfected with miR181a mimic in cells resulted in the decreased expression of $\mathrm{Bcl}-2$. The alteration of miR-181a expression did not significantly affect the chemosensitivity to adriamycin in MCF-7 and MCF7/ADR cells with genetic knockout of $\mathrm{Bcl}-2$. miR-181a may suppress $\mathrm{Bcl}-2$ expression by forming imperfect base pairing with the $3^{\prime} \mathrm{UTR}$ of $\mathrm{BCl}-2$ gene such that a negative relationship between miR-181a and $\mathrm{BCl}-2$ in MCF-7 and MCF-7/ADR cells is observed. Conclusions: At least in part, the detection of miR-181a may direct the clinical medication in patients with neoadjuvant chemotherapy because of miR-181a enhanced adriamycin-induced apoptosis via targeting $\mathrm{BCl}-2$.
\end{abstract}




\section{Introduction}

Breast cancer (BC) is a health-threatening disease of great magnitude among women around the world. Breast cancer-related outcomes, including an increasing incidence and decreasing mortality, are due to effectively early diagnosis and the standardized existing treatments [1]. Traditionally, a definitive surgical procedure is one of the fundamental treatment in BC. Few patients present with unresectable, locally advanced disease and require the systemic treatment $[2,3]$. Thus, designated chemotherapy is used to diminish the volume of tumor or prolong the survival period of these patients.

Currently, several microRNAs have been investigated for their expression in tumourigenesis to discover the possible role in pathogenesis and evaluate their use for diagnosis and prognosis of some cancers [4-8]. Only a few of studies [9-16] are investigating the involvement of miR-181a in chemosensitivity in various disorders. B. Zhou et al. showed that miR-181a regulated SIRT1 and improved hepatic insulin sensitivity. Decreased miR181a might be a potential new strategy for treating insulin resistance and type 2 diabetes [9]. It aslo facilitated Growth Factor Signaling in tamoxifen-resistant breast cancer by downregulating TIMP3 [14]. Consistent with our hypothesis, miR-181a sensitized K562/A02 and SGC7901/VCR cell lines by targeting Bcl-2 $[15,16]$. Curretly, 28 target genes of hsa-miR181a have been validated $[10-12,17,18]$. The presence of many matching sites between the mature miR-181a seed region have facilitated the role of miR-181a in chemotherapy, giving them access to the modulation of a huge number of cellular pathways, especially apoptosis or anti-apoptosis ones.

MCF-7(ER+, PgR+,HER2-) is one of the most representative low-invasive breast cancer cells treatment by adriamycin. Our study identified that miR-181a played an important role in chemosensitivity to adriamycin in MCF-7 and MCF-7/ADR cells via targeting Bcl-2. miR-181a-based therapy might be a potential new strategy for low-invasive breast cancer patients with poor-response to adriamycin.

\section{Materials and Methods}

\section{Cell Culture}

Human breast caner cell line MCF-7 and its resistant MCF-7/ADR were obtained from ATCC (Rockville, MD). Cells were cultured in DMEM supplemented (Gibco, Grand Island, NY, USA) with 10\% fetal bovine serum (Gibco) in a humidified atmosphere of $5 \% \mathrm{CO}_{2}$ at $37^{\circ} \mathrm{C}$.

\section{Cell transfection}

Hsa-miR-181a mimic, hsa-miR-181a inhibitor and hsa-miR negative control were synthesized by Genepharma (Shanghai, China, showed in Table 1). Small inhibitory RNA (siRNA) to Bcl-2 and SignalSilence ${ }^{\circledR}$ Control siRNA were purchased from Cell Signaling Technology. Twenty-four hours prior to transfection, MCF-7 and MCF-7/ADR cells were plated in 6-well plates $\left(2.5 \times 10^{5}\right.$ cell/well) and then transfected with miR-181a mimic (50nM) or miR-181a inhibitor (50nM) or miR negative control (50nM) using Lipo2000 (Invitrogen, Carlsbad, CA). $100 \mathrm{nM}$ SignalSilence ${ }^{\circledR}$ Control siRNA or SignalSilence $®$ Bcl-2 siRNA were transfected into cells following the manufacturer's protocol.

\section{Cell Survival Analysis}

Twenty four hour post-transfection, cells were seeded into 96 -well plates $\left(6 \times 10^{3} /\right.$ well $)$ and treated with ADR (Hisun Pharmaceutical Co., ZheJiang, China) at different concentration for $48 \mathrm{~h}$. The Cell Counting Kit-8 assay (Dojindo, Kumamoto Prefecture, Kyushu, Japan) was used to determine relative cell growth according to the manufacturer's instructions. Data shown are representative of three independent experiments.

Apoptosis assay

MCF-7/ADR and MCF-7 cells were transfected with miR-181a mimic and miR-181a inhibitor for 48 has decribed above and incubated with $20 \mathrm{mg} / \mathrm{L}$ ADR or $0.2 \mathrm{mg} / \mathrm{L}$ ADR for 48 hours, respectively. Then, 


\begin{tabular}{|c|c|c|}
\hline Cellular Phys & Cell Physiol Biochem 2013;32:1225-1237 & \\
\hline and Biochemistry & $\begin{array}{l}\text { DOI: } 10.1159 / 000354521 \\
\text { Publisnea onine: November 14, } 2013\end{array}$ & $\begin{array}{l}\text { O } 2013 \text { S. Karger AG, Basel } \\
\text { www.karger.com/cpb }\end{array}$ \\
\hline
\end{tabular}

Table 1. The sequence of materials

\begin{tabular}{ll}
\hline Material & Sequence (5'-3') \\
\hline Bcl-2 Forward & GGTGGGGTCATGTGTGTGG \\
Bcl-2 Reverse & CGGTTCAGGTACTCAGTCATCC \\
$\beta$-actin Forward & TTCTACAATGAGCTGCGTGTG \\
$\beta$-actin Reverse & CAGCCTGGATAGCAACGTACA \\
hsa-miR negative control & UUCUCCGAACGUGUCACGUTT \\
hsa-miR-181a mimic & AACAUUCAACGCUGUCGGUGAGU \\
hsa-miR-181a inhibitor & ACUCACCGACAGCGUUGAAUGUU \\
\hline
\end{tabular}

$1 \times 10^{6}$ cells were collected and washed twice with ice-cold PBS. Cells were dual stained using FITC Annexin $\mathrm{V}$ apoptosis Detection Kit I (BD Biosciences, Franklin Lakes, NJ) according to the manufacturer's protocol. Stained cells were immediately analyzed using a flow cytometer (BD Biosciences).

\section{Real-time PCR assays}

Total RNA was extracted by Trizol ( Invitrogen, Carlsbad, CA) and subjected to reverse transcription using TaqMan ${ }^{\circledR}$ MicroRNA Reverse Transcription Kit (Applied Biosystems, Foster City, CA) according to the manufacturer's instructions. For detection of mature miRNAs, the TaqMan ${ }^{\circledR}$ microRNA Assay (Applied Biosystems, Foster City, CA) was used according to the manufacturer's instructions. The quantitative analysis of the change in expression levels was calculated by ABI 7300 real-time PCR machine (Applied Biosystems, Carlsbad, CA). An average of three experiments each performed in triplicate with standard errors is presented. Primer quality was analyzed by dissociation curves. Primer sequences are listed in Table 1. Data were analyzed by comparing Ct values.

\section{miRNA Target Gene Identification}

For miRNA target gene identification, TargetScan (http://www.targetscan.org) and miRecord (http:// mirecords.biolead.org) were used to identify target genes of identified miRNAs.

\section{Dual Luciferase Activity Assay}

The 3'UTR of human Bcl-2 cDNA containing the putative target site for the mature miR-181a was chemically synthesized and inserted at the XbaI site. MCF-7 cells $\left(6 \times 10^{4} /\right.$ well $)$ were incubated in 24well plates and co-transfected with miR-181a mimic or control mimic and PGL3-Bcl-2 3'UTR-WT vector or PGL3-Bcl-2 3'UTR-MUT vector containing firefly luciferase reporter gene and 3'UTR of Bcl-2 gene (Promega, Madison, WI) using lipo2000 (Invitrogen, Carlsbad, CA). Luciferase activity was measured 48 hours after transfecion using the Dual Luciferase Reporter Assay System (Promega) and normalized to renilla luciferase activity.

\section{Western blot}

Cells were extracted and prepared in modified RIPA buffer (Beyotime, Jiangsu, China). Proteins were separated by $10 \%$ SDS-PAGE, transferred to polyvinylene difluoride membranes (Bio-Rad, Hercules, CA) and incubated with primary antibodies against human Bcl-2 (1:500, Santa Cruz, CA) overnight at $4^{\circ} \mathrm{C}$. The horseradish peroxidase-conjugated secondary antibody were from Dako Co, Danmark. Immunoreactive bands were visualized by chemiluminescence with pierce ECL detection reagent (Millipore, Billerica, MA). $\beta$-actin (1:4000, Bioworld, MN) was used as an internal loading control. Shown are representative data from individual experiments that were repeated at least twice.

\section{Patients and Samples}

Samples were obtained from patients treated at the Jiangsu Cancer Hospital, Affiliated Hospital of Nanjing Medical University, from 2010-2012, comprising neoadjuvant chemotherapy (NAC) patients $(n=35)$ and non-neoadjuvant chemotherapy patients $(n=43)$ with non-metastic disease at diagnosis. The 
Table 2. Comparison of several clinicopathologic parameters and expression levels of miR-181a in Breast Cancer

\begin{tabular}{|c|c|c|}
\hline & $\mathrm{N}$ & miR-181a ${ }^{a}$ \\
\hline \multicolumn{3}{|l|}{ Age } \\
\hline$<47$ & 21 & $-3.501(-5.964--1.881)$ \\
\hline$>47$ & 22 & $-2.735(-4.124--0.930)$ \\
\hline$P$ value ${ }^{b}$ & & $>0.05$ \\
\hline \multicolumn{3}{|l|}{ Histology } \\
\hline invasive dutal carcinoma & 35 & $-3.037(-5.581--0.998)$ \\
\hline invasive lobular carcinoma & 8 & $-2.745(-4.571--2.024)$ \\
\hline P valueb & & $>0.05$ \\
\hline \multicolumn{3}{|l|}{ Clincal stage } \\
\hline I & 34 & $-3.037(-5.964--0.994)$ \\
\hline II & 9 & $-2.595(-4.094--1.171)$ \\
\hline$P$ valueb & & $>0.05$ \\
\hline \multicolumn{3}{|l|}{ Tumor diameter } \\
\hline $\mathrm{T}<2 \mathrm{~cm}$ & 14 & $-3.037(-6.629--0.604)$ \\
\hline $\mathrm{T}>2 \mathrm{~cm}$ & 29 & $-2.992(-4.395--1.224)$ \\
\hline P value ${ }^{b}$ & & $>0.05$ \\
\hline \multicolumn{3}{|l|}{ Lymph node } \\
\hline Negative & 24 & $-2.199(-4.049--0.565)$ \\
\hline Positive & 19 & $-3.138(-8.224--1.746)$ \\
\hline P valueb & & $>0.05$ \\
\hline \multicolumn{3}{|l|}{ Her-2 } \\
\hline Negative & 18 & $-2.957(-4.596--0.909)$ \\
\hline Positive & 25 & $-3.806(-5.964--2.099)$ \\
\hline P value ${ }^{b}$ & & $>0.05$ \\
\hline \multicolumn{3}{|l|}{ Ki-67 } \\
\hline Negative & 19 & $-2.417(-5.391-0.65)$ \\
\hline Positive & 24 & $-2.921(-4.541--1.29)$ \\
\hline$P$ value ${ }^{b}$ & & $>0.05$ \\
\hline $\begin{array}{l}\text { aMedian of relative ex } \\
\text { parenthesis( normalized } \\
\text { between two groups }\end{array}$ & & $\begin{array}{l}\text { th } 25 \text { th-75th percentile in } \\
\text { scale); bMann-Whitney U test }\end{array}$ \\
\hline
\end{tabular}

diagnosis of each case was confirmed independently by two pathologists based on WHO classification. In accordance with the following criteria: These NAC patients were to receive 2-6 cycles of preoperative neoadjuvant chemotherapy with FAC or AC (ADR $40 \mathrm{mg} / \mathrm{m}^{2}$ and CTX $0.6 \mathrm{~g} / \mathrm{m}^{2}$ combined with or without $5-\mathrm{Fu} 0.5 \mathrm{~g} / \mathrm{m}^{2}$ ) on day 1 with treatments repeated every 21 days; a histological diagnosis of invasive ductal or lobular carcinoma with at least one measurable lesion; a clinical stage of IIA-IIB; without triple negative breast cancer (TNBC) or HER-2 positive patients. According to the patient's response assessed by computer tomography (CT) tests and detection of serum tumor marker. Tissue samples of NAC patients were classified into responders (complete reponse or partial reponse) and non-responders (stable disease or progressive disease). Therapy of other forty-three non-neoadjuvant chemotherapy patients consisted of breast conserving surgery or modified radical mastectomy without neoadjuvant therapy. The patients gave written informed consent in accordance with the Declaration of Helsinki and the study was approved by the Ethics Committee of the Jiangsu Cancer Hospital. 
Fig. 1. Expression of miR181a and Bcl-2 in MCF-7 and MCF-7/ADR Breast Cancer Cells. (A) $\mathrm{IC}_{50}$ of ADR in MCF-7 and MCF-7/ADR cells was $0.36 \pm 0.013$ and $64.07 \pm 2.074 \mathrm{mg} / \mathrm{L}$, respectively (mean \pm SD; Kruskalwallis test; $\mathrm{p}<0.05) . \quad(\mathrm{B}, \mathrm{C})$ The relative expression levels of miR-181a and Bcl-2 mRNA were performed by qRT-PCR in MCF-7 and MCF7/ADR (Kruskal-wallis test; $\mathrm{p}<0.05$ ). (D) Compared to MCF-7 cells, Bcl-2 protein expression was markablely increased in MCF-7/ADR. All experiments were performed in triplicate.
A

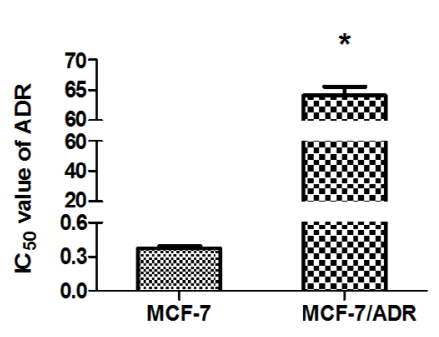

C

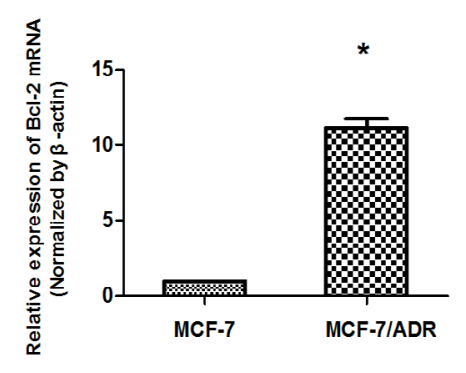

B

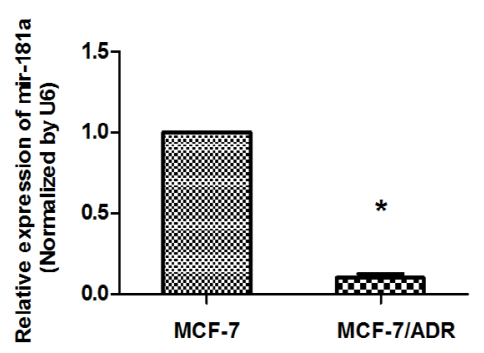

D

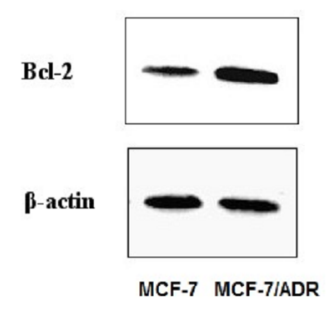

Statistical Analysis

The results presented are average of at least three experiments each performed in triplicate with standard errors. Statistical analyses were performed by analysis of variance, followed by Kruskal-wallis test or Spearman rank test, using SPSS 20.0 statistical package. All test were two tailed; $\mathrm{p}<0.05$ were considered significant and are indicated with asterisks.

\section{Results}

Expression of miR-181a in human breast cancer specimens

The aberrant expression in breast cancer prompted us to explore the further possible biological significance of miR-181a in tumorigenesis. To explore whether miR-181a were associated with several clinicopathologic parameters, the expression of miR-181a in 43 patients treated without neoadjuvant therapy were detected. The clinical and pathological characteristics of these patients are summarized in Table 2. Detailed backgrounds for each patient, including age, histology, clinical staging, tumor diameter, lymph node, expression levels of Her-2 and Ki-67 have been collected. Analysis of these data showed that there was no association between the clinicopathological features and miR-181a $(p>0.05$, Table 2$)$.

\section{Negative Correlation Expression of miR-181a and Bcl-2 in MCF-7 and MCF-7/ADR Breast} Cancer Cells

MCF-7 and MCF-7/ADR cells were exposed to ADR at different concentrations for 48 hours. As shown in Fig. $1 \mathrm{~A}$, the half maximal inhibitory concentration $\left(\mathrm{IC}_{50}\right.$ ) of ADR in MCF7 and MCF-7/ADR cells was $0.36 \pm 0.01$ and $64.07 \pm 2.07 \mathrm{mg} / \mathrm{L}(\mathrm{mean} \pm \mathrm{SD})$, respectively. To further investigate whether there was any difference of miR-181a expression between MCF7 and MCF/ADR cell line, the results showed that the relative expression level of miR-181a significantly down-regulated in MCF-7/ADR, compared with its parental MCF-7 cell line(Fig. 1B; MCF-7 vs. MCF-7/ADR: 1-fold vs. 0.10-fold \pm 0.03 , mean $\pm S D, p<0.05$ ). According to computational analysis by TargetScan and miRecord, Bcl-2 was chosen for further validation of its probable relationship with miR-181a. In MCF-7/ADR, Bcl-2 mRNA expression (11.14- 
Fig. 2. qRT-PCR was performed to detect the transfection efficiency of miR-181a mimic and inhibitor in MCF-7 and MCF-7/ADR cells. (A) At 48 hours after transfection, miR181a expression was upregulated in mimic groups and downregulated in inhibitor group as compared to negative control in MCF-7 and MCF-7/ADR cells [MCF-7 cell: untreated cell, 1-fold vs. mimic, 8.95-fold $\pm 0.62(\mathrm{p}=0.000)$ vs. control mimic, 1.02 -fold \pm 0.03 vs. inhibitor, 0.08 -fold $\pm 0.04(\mathrm{p}=0.000)$ vs. control inhibitor, 1.05 -fold \pm 0.04 , mean $\pm S D$; MCF-7/ADR cell: untreated cell, 1 -fold vs. mimic, 6.87 -fold $\pm 0.43(\mathrm{p}=0.000)$ vs. control mimic, 1.01 -fold \pm 0.04 vs. inhibitor, $\quad 0.17$-fold $\pm 0.05(\mathrm{p}=0.000)$ vs. control inhibitor, 0.99-fold \pm 0.02 , mean $\pm S D]$. $(B, C)$ After transfection, the survival rates of MCF-7 and MCF7/ADR treated with different concentrations of ADR for $48 \mathrm{~h}$ were tested by cck- 8 assay.

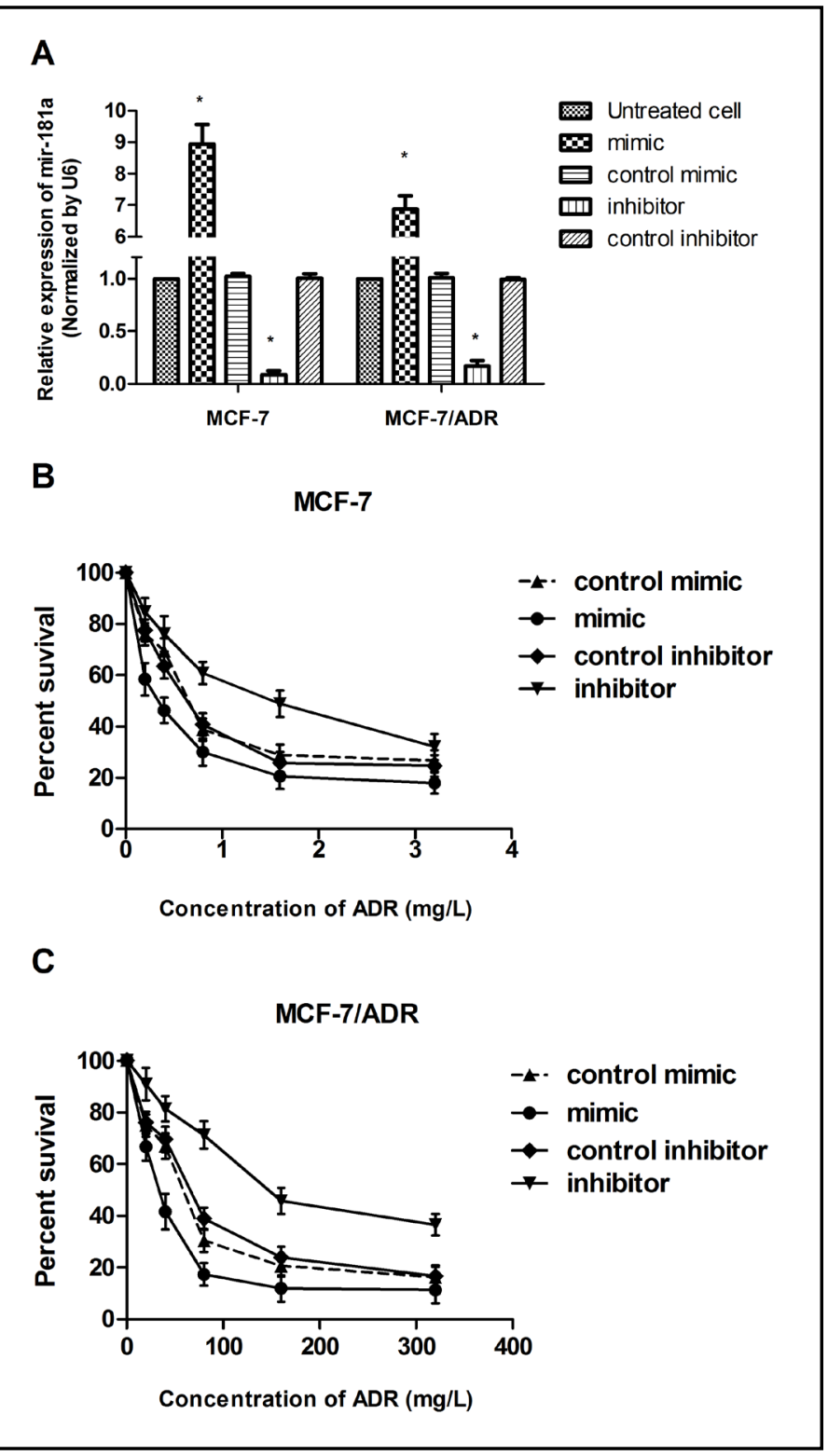

B

C

fold \pm 0.84 , mean \pm SD) and protein expression (1.96-fold \pm 0.43 , mean \pm SD) were both marked increased, compared with MCF-7 cell line (p<0.05; Fig. 1C, 1D).

The alteration of miR-181 a expression involved in chemosensitization to adriamycin

To determine the effect of miR-181a expression on chemosensitivity to adriamycinbased chemotherapy, miR-181 mimic or miR-181a inhibitor or miR negative control were sucessfully transfected into MCF-7 and MCF-7/ADR cells. At 48 hours after transfection, expression of miR-181a was determined by quantitative RT-PCR assay (Fig. 2A). Detected by cck- 8 assay, transfection with the miR-181a inhibitor reduced susceptibility to ADR. Conversely, cells transfected with miR-181a mimic showed the effect of chemosensitization. (Fig. 2B, 2C).

$B c l-2$ is required for the effect of miR-181a on chemosensitivity

To investigate whether Bcl-2 directly regulates chemosensitivity to adramycin, Bcl2 siRNA was transfected into MCF-7 and MCF-7/ADR cell lines. The results showed that genetic knockout of Bcl-2 attenuated resistance to ADR in both cell lines [Bcl-2 expression: 
Fig. 3. In MCF-7 and MCF-7/ADR cells transfected with Bcl-2 siRNA, the IC50 value were $0.21 \pm 0.04 \mathrm{mg} / \mathrm{l}$ and $\quad 44.39 \pm 1.65 \mathrm{mg} / \mathrm{l}($ mean $\pm \mathrm{SD})$, respectively( $\mathrm{p}<0.05)$ (Fig. 3A). Compared with cells transfected with Bcl-2 siRNA only, the alteration of miR-181a expression didn't obviously enhance or reduce the chemosensitivity to adriamycin in MCF-7 and MCF-7/ADR cells transfected with Bcl-2 siRNA and miR-181a mimic/ inhibitor( $p>0.05$ )(Fig. 3B, 3C).

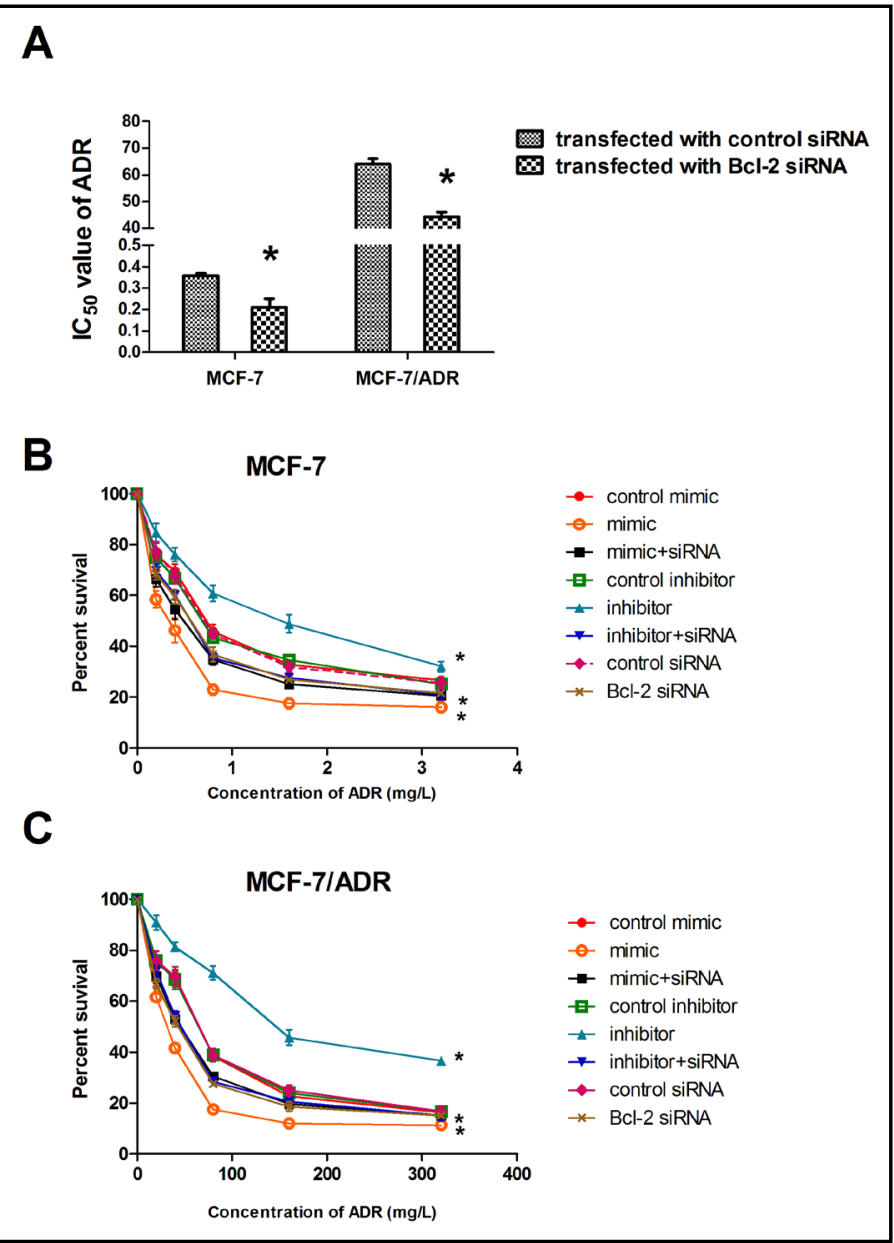

MCF-7-control siRNA, 1-fold vs. MCF-7-Bcl-2 siRNA, 0.21-fold $\pm 0.09(\mathrm{p}=0.000)$; MCF-7/ ADR-control siRNA, 1-fold vs. MCF-7/ADR-Bcl-2 siRNA, 0.25-fold $\pm 0.07(\mathrm{p}=0.000)]$. In MCF7 and MCF-7/ADR cells transfected with Bcl-2 siRNA, the IC50 value were $0.21 \pm 0.04 \mathrm{mg} / \mathrm{l}$ and $44.39 \pm 1.65 \mathrm{mg} / \mathrm{l}($ mean $\pm \mathrm{SD})$, respectively(Fig. 3A). Compared with cells transfected with Bcl-2 siRNA only, the alteration of miR-181a expression didn't obviously enhance or reduce the chemosensitivity to adriamycin in MCF-7 and MCF-7/ADR cells transfected with Bcl-2 siRNA and miR-181a mimic/inhibitor, which suggesting that miR-181a regulate the chemosensitivity to adriamycin mainly through Bcl-2 but not other genes(Fig. 3B, 3C).

Downregulation of miR-181a decreased adriamycin-induced apoptosis of MCF-7 and MCF7/ADR

Compared with the negative controls, the apoptosis rate was significantly increased in MCF-7 and MCF-7/ADR cells transfected with miR-181a mimic [Fig. 4A; apoptosis rate in MCF-7/ADR cells: control , $5.3 \% \pm 0.41 \%$ vs. mimic , $9.7 \% \pm 1.17 \%(\mathrm{p}<0.05)$ vs. control mimic $+A D R, 15.2 \% \pm 0.93 \%(\mathrm{p}<0.05)$ vs. mimic + ADR, $27.7 \% \pm 1.30 \%(\mathrm{p}<0.05)$, mean $\pm S D]$. Furthermore, downregulation of endogenous miR-181a inhibited ADR-induced apoptosis in cells [Fig. 4B; apoptosis rate in MCF-7 cells: control , $4.9 \% \pm 0.33 \%$ vs. inhibitor , $1.7 \% \pm 0.40 \%$ $(\mathrm{p}<0.05)$ vs. control inhibitor $+A D R, 15.1 \% \pm 1.92 \%(\mathrm{p}<0.05)$ vs. inhibitor $+A D R, 4.8 \% \pm 0.67 \%$ $(\mathrm{p}<0.05)$, mean $\pm S D]$.

Evidence of inverse regulation of Bcl-2 by miR-181a

To understand the mechanism by which miR-181a modulated chemosensitivity of MCF7 cell line, two prediction algorithms, TargetScan and miRecord, were utilized to identify the 
A MCF-7/ADR

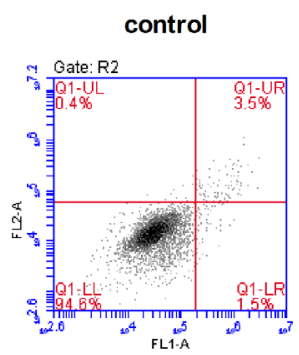

control mimic+ADR

mimic

mimic+ADR

B MCF-7
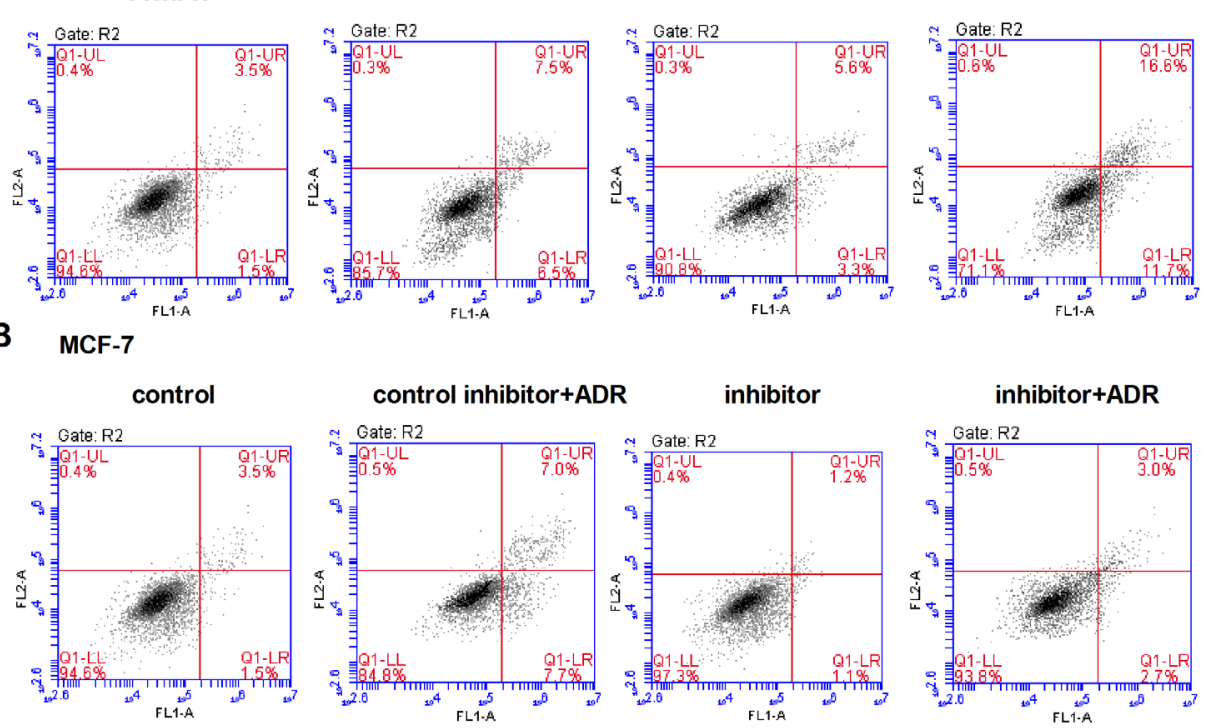

C
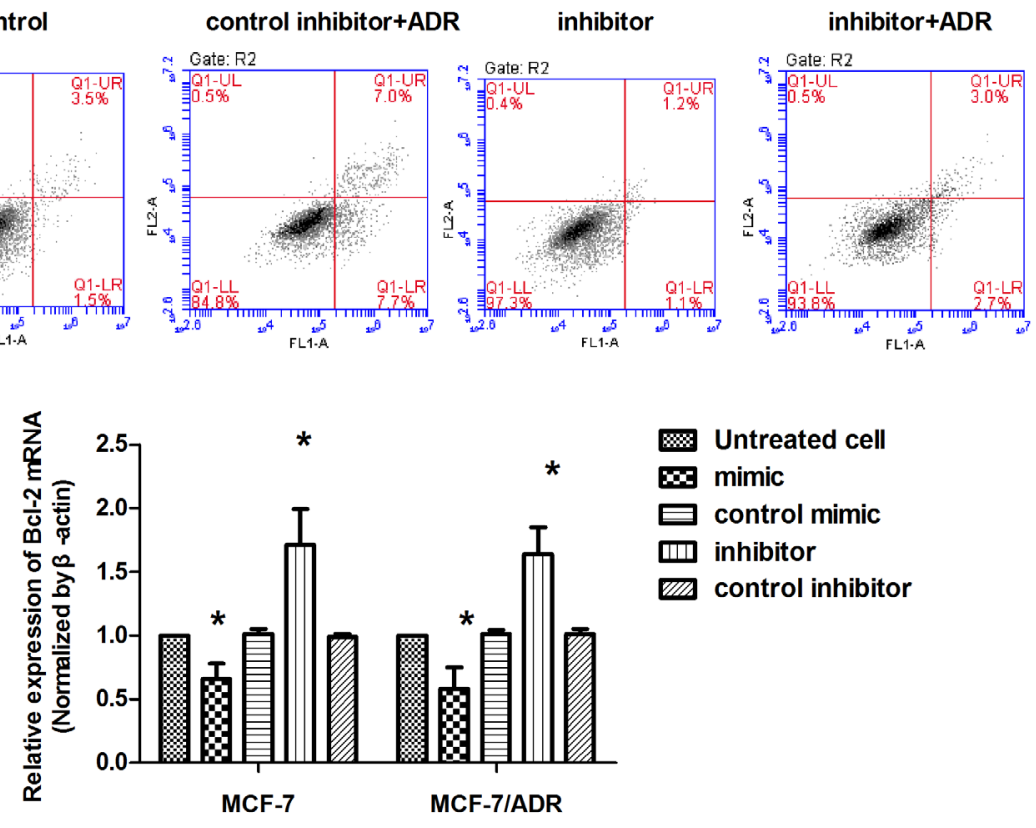

D

MCF-7/ADR

E

MCF-7

Bcl-2

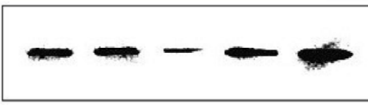

$\beta$-actin
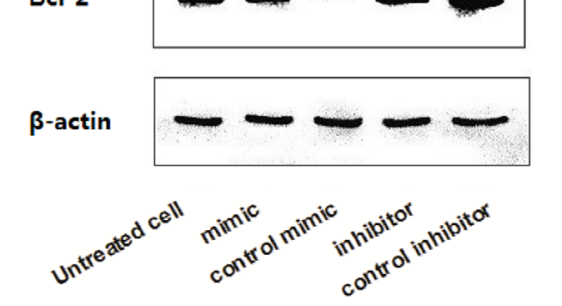

Untreated cell

$\mathbb{B}$ mimic

$\square$ control mimic

四 inhibitor

m control inhibitor
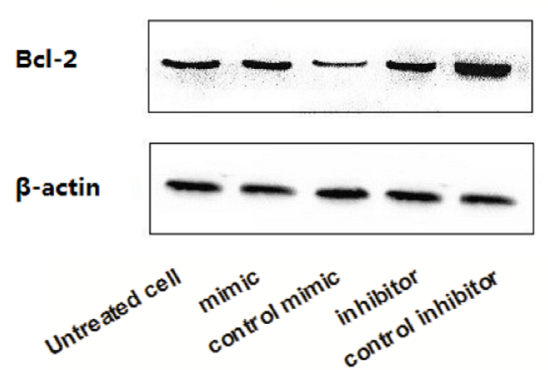

Fig. 4. ADR-induced apoptotic cells induced in MCF-7 and MCF-7/ADR cells transected with miR-181a inhibitor, mimic and their negative controls, respectively. (A) By flow cytometry analysis, overexpression of miR-181a induced apoptosis in MCF-7/ADR cells compared with the negative control. (B) Downregulation of endogenous miR-181a inhibited ADR-induced apoptosis in MCF-7 cells.

potential target gene. Bcl-2, whose gene product is known to have a role in reducing apoptosis, was one of the possible target genes of miR-181a. Thus, qRT-PCR and Western blot were performed to test whether expression of Bcl-2 could be altered by miR-181a. As shown in Fig. $5 \mathrm{~A}$, the assay revealed that the decreased expression of Bcl-2 mRNA in MCF-7 cells was altered 


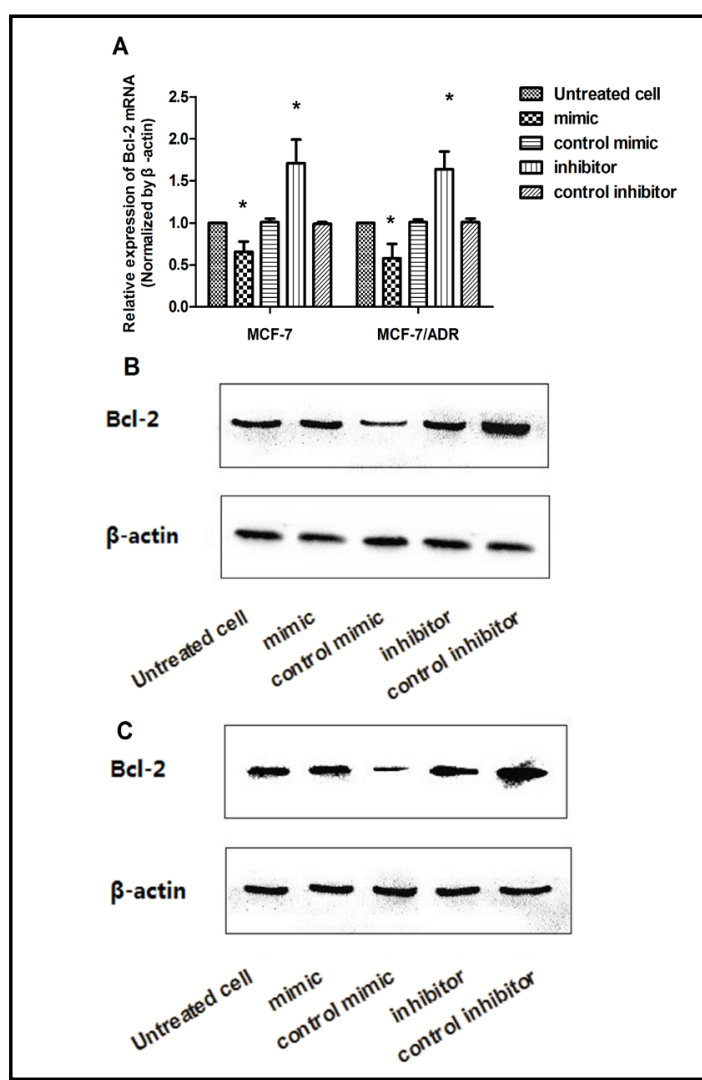

Fig. 5. The expression of Bcl-2 mRNA and protein levels in MCF-7 and MCF-7/ADR cell lines transfected with miR-181a mimic or inhibitor. $(A, B, C)$ The miR181a inhibitor increased the expression of $\mathrm{Bcl}-2$ mRNA and protein levels in both MFC-7 and MCF-7/ ADR. Meanwhile, transfected with miR-181a mimic in MCF-7 and MCF-7/ADR cells resulted in a significant reduction of $\mathrm{Bcl}-2$ expression both in mRNA and protein expression $(A, B, C)$.
A

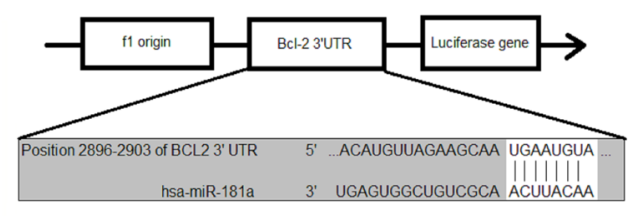

B

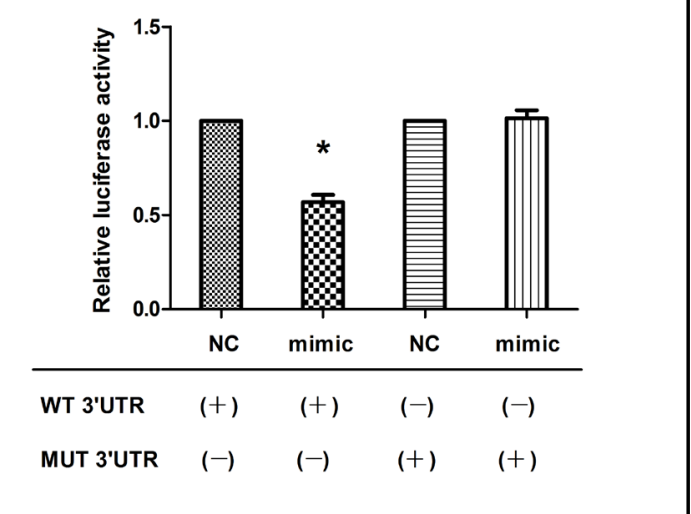

Fig. 6. miR-181a inversely regulates the expression of Bcl-2 via targeting its 3'-UTR. (A) The schematic representation of the PGL3-Bcl-2 3'UTR-WT vector and the complementary site of miR-181a in Bcl-2 3'UTR highlighted in white. (B) Compared to the negative control, miR-181a repressed the activity of luciferase fused to the WT Bcl-2 3'UTR, resulting in a $45 \%$ reducion ( Kruskal-wallis test; $\mathrm{p}<0.05$ ), while it failed to repress the mutated one (Kruskal-wallis test; $p>0.05$ ). Data are representative of three independent experiments.

by the overexpression of miR-181a [untreated cell, 1 -fold vs. mimic, 0.66 -fold $\pm 0.12(p=0.000)$ vs. control mimic, 1.01 -fold \pm 0.04 vs. inhibitor, 1.71 -fold $\pm 0.28(\mathrm{p}=0.000)$ vs. control inhibitor, 1.00 -fold \pm 0.02 , mean $\pm \mathrm{SD}$ ] and transfected with miR-181a inhibitor increased the expression of Bcl-2 mRNA [untreated cell, 1 -fold vs. mimic, 0.58 -fold $\pm 0.17(\mathrm{p}=0.000)$ vs. control mimic, 1.01 -fold \pm 0.03 vs. inhibitor, 1.63 -fold $\pm 0.21(\mathrm{p}=0.000$ ) vs. control inhibitor, 1.01 -fold \pm 0.04 , mean $\pm \mathrm{SD}$ ], consistent with the protein expression tested by western blot (Fig. 4B, 4C; $\mathrm{p}<0.05$ ).

Computational analysis informed that there was a binding site on the 3'UTR of the Bcl-2 for miR-181a, which was highly conserved in mammals. To ascertain whether these miR181a-binding sequences directly contributed to the negative regulation of Bcl-2 expression, we explored the effects of miR-181a on activity of the target gene using the vectors that either contained wild-type or mutant miR-181a-binding sequences. As shown in Fig. 6, miR181a repressed the activity of luciferase fused to the WT Bcl-2 3'UTR, resulting in a $45 \%$ reduction $(\mathrm{p}<0.05)$, while it failed to surpress the mutated one $(\mathrm{p}>0.05)$. It indicated that miR-181a may suppress Bcl-2 expression by forming imperfect base pairing with the 3'UTR of Bcl-2 gene. 
A

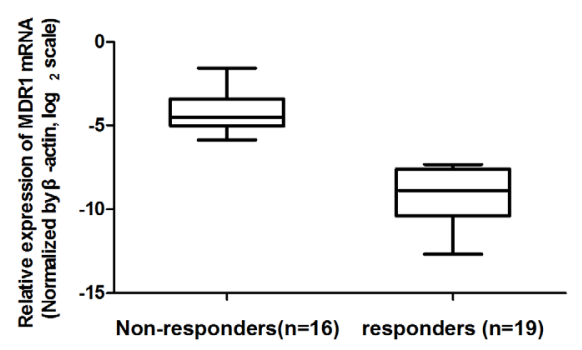

C

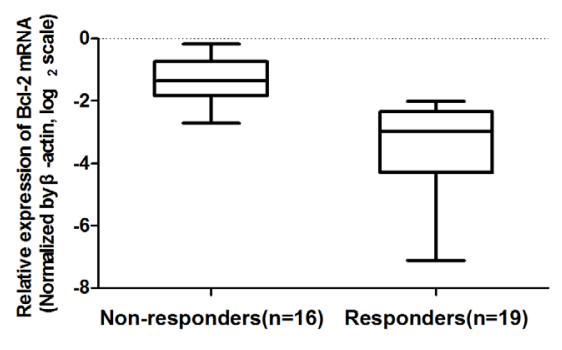

B

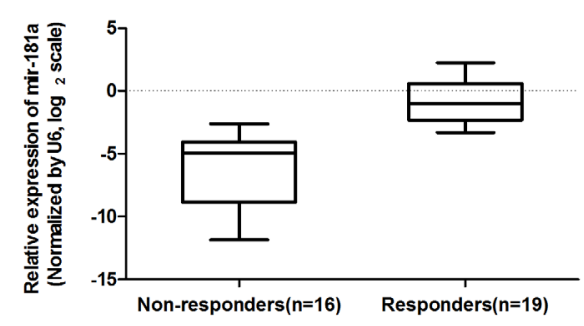

D

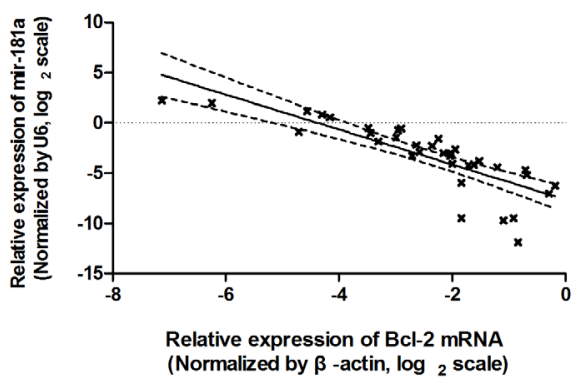

Fig. 7. Negative Correlation of miR-181a and Bcl-2 in Breast Cancer Patients with Adriamycin-based Neoadjuvant Chemotherapy. (A) The MDR1 mRNA expression was significantly elevated in non-responders. miR-181a (B) and Bcl-2 (C) expression were detected in responders and non-responders by qRT-PCR. (D) The inverse correlation bewteen miR-181a and Bcl-2 in breast specimens was analyzed by liner regression analysis (Spearman rank test; rho=-0.81; $<<0.05$, two-tailed).

miR-181a and Bcl-2 mRNA expression in Breast Cancer Patients with Adriamycin-based Neoadjuvant Chemotherapy

For further evaluate the correlation between the expression of miR-181a and Bcl2 mRNA in Breast Cancer patients, 35 selected patients with neoadjuvant chemotherapy were classified into responders $(n=19)$ and non-responders $(n=16)$ according to RECIST criteria. Three patients with a complete response (CR) and sixteen patients with a partial response (PR) were regarded as clinical responders. Eight patients had progressive disease (PD), and eight patients had stable disease (SD). Those with SD or PD were deemed to be non-responders. The expression of MDR1 mRNA should be detected by qRT-PCR in the non-responders and responders. The MDR1 mRNA expression was significantly elevated in non-responders $(\mathrm{n}=16)$ (Fig. $7 \mathrm{~A}$; non-responders vs. responders: $4.10 \pm 1.28$ vs. $9.07 \pm 1.52$, mean $\pm S D, p<0.05$; ), with a negative correlation with ADR sensitivity (Spearman rank test; rho=-0.90; $<<0.05$, two-tailed). To verify the hypothesis that there is a potential relevance bewteen the expression of miR-181a and chemosensitivity, miR-181a and Bcl-2 mRNA expression was evaluated by qRT-PCR in 35 breast tumors . Consequently, linear regression analysis was used to reveal that miR-181a expression was down-regulated in non-responders group ( $\mathrm{p}<0.05$; Fig. 7B), with a inverse correlation with Bcl-2 mRNA expression (Spearman rank test; rho=-0.81; $\mathrm{p}<0.05$; Fig. 7C, 7D). Considering the well-characterized role of Bcl-2 in anti-apoptosis, it suggested that in some case, miR-181a play a role in poor response to adriamycin-based neoadjuvant chemotherapy in breast cancer patients via targeting Bcl-2. 


\section{Discussion}

miR-181a is one of the crucial means important microRNAs in cellular differentiation and development, evidenced by its dysregulation in autoimmunity and cancers $[19,20]$. It widely exists in human organs, with its highest abundance in brain, liver and eyes during early development [21]. miR-181a was aslo abundantly expressed in the Tymphocyte and B lymphoid cell [22]. It is still controversial that miR-181a acts as a oncomir or a tumor suppressor. The global downregulation of miR-181a was observed in most soild tumors [17, 18, 23-26] and chronic lymphocytic leukaemia (CLL) [27]. On the contrary, the role of miR181a in breast cancer was confirmed recently. It was documented that the upregulation of miR-181a might contribute to the development of cancer stem cell properties to promote the metastasis and invasion [28].

The miR-181 family consists of four members (miR-181a,b,c,d) whose seed region is complementary to a large range of identified mRNAs. miR-181a is able to modulate its target genes by inhibitory binding or accelerating degradation of mRNA [29]. Bcl-2, as one of its target mRNAs, inhibits mitochondrial metabolism and adriamycin-induced apoptosis in MCF-7 cells. It might prevent the early apoptotic events and revealed post-mitotic multinucleation without affecting cell cycle arreast [30,31]. Once the withdrawal, Bcl-2 protein could enhance the capacity of proliferation in tumor cells [15]. In the our study, we identified that the level of miR-181a expression in MCF-7/ADR cells was significantly lower than that in MCF-7 cells by qRT-PCR. The results aslo showed that genetic knockout of Bcl-2 attenuated resistance to ADR in both cell lines, miR-181a regulated the chemosensitivity to adriamycin mainly through Bcl-2 in MCF-7 and MCF-7/ADR cells. To further investigated the molecular mechanism of how miR-181a invovled in chemosensitivity to ADR in MCF-7 and MCF-7/ADR cells, we transfected cells with miR-181a mimic or inhibitor. The results showed that downregulation of miR-181a reduced susceptibility to ADR and inhibited ADRinduced apoptosis in cells. On the contrary, miR-181a mimic could enhance apoptosis of ADR-induced MCF-7/ADR cells detected by flow cytometry. Moreover, dual luciferase activity assay was performed to determine that overexpression of miR-181a suppressed Bcl-2 gene through binding to motifs in the 3'UTR complementary to the seed region. Concurrent with poor response to ADR, decreased miR-181a and aberrant upregulation of Bcl-2 mRNA were observed in non-responders group, compared with the 19 responders. These data indicated that miR-181a may serve as a potential biomarker to predict the response to ADRbased neoadjuvant chemotherapy. Our conclusion is supported by both vitro and clinical investigation.

Prior to neoadjuvant chemtherapy, all patients received core needle biopsy to confirm the nature and pathological type of breast tumor. In the future, based on our findings, we could detect the expression of miR-181a in biopsy specimens to speculate the effect of ADRbased neoadjuvant chemotherapy. Our study provided a theoretical basis for the choice of medicine.

Additionally, we demonstrated that there was no association between the clinicopathological features and miR-181a. In a recent study by Yang et al. [32], patients with higher level of miR-181a was more likely to have lymph node metastases. This discrepancy may be due to cell type-specific response to miR-181a. It is advisable to regard its function in a complete setting that is the context in which it is undertaking.

However, the exact mechanism in mediating tumor cells response to chemotherapy is still unknown. We discovered that MDR1 mRNA detected by qRT-PCR was generally elevated in those patients which were classified into non-responders. These data of MDR1 were aslo in agreement with our classification according to the CT tests and detection of serum tumor marker. As a bold assumption, miR-181a may aslo have an intersecting complex effect that spans a multiplicity of drug-resistance pathways and processes. It needs to be further investigated.

In summary, the study first identified that there was a causal correlation between overexpression of miR-181a and chemosensitivity to adriamycin in both vitro and NAC 
patients via targeting Bcl-2. According to the level of miR-181a, we may evaluate whether the patient is sensitive to ADR-based neoadjuvant chemotherapy. It may direct the clinical medication. miiR-181a-based therapy may serve as a highly promising option for patients with poor-response to adriamycin because of its enhanced adriamycin-induced apoptosis. Owing to the complex networks among miR-181a and its targets, a better understanding of miRNA-based therapy requires further investigation before designing safe procedure.

\section{Conflict of Interests}

The authors have declared that no competing interests exist.

\section{Acknowledgements}

This work was supported by grants from the National Natural Science Foundation of China (81272470). The funders had no role in study design, data collection and analysis, decision to publish, or preparation of the manuscript.

\section{Reference}

1 Berry DA, Cronin KA, Plevritis SK, Fryback DG, Clarke L, Zelen M, Mandelblatt JS, Yakovlev AY, Habbema JD, Feuer EJ: Effect of screening and adjuvant therapy on mortality from breast cancer. New Engl J Med 2005;353:1784-1792.

2 Craft BS, Hortobagyi GN, Moulder SL: Adjuvant biologic therapy for breast cancer. Cancer J 2007;13:156161.

-3 Harvey JM, Clark GM, Osborne CK, Allred DC: Estrogen receptor status by immunohistochemistry is superior to the ligand-binding assay for predicting response to adjuvant endocrine therapy in breast cancer. J Clin Oncol 1999;17:1474-1474.

-4 Iorio MV, Ferracin M, Liu CG, Veronese A, Spizzo R, Sabbioni S, Magri E, Pedriali M, Fabbri M, Campiglio M, Ménard S, Palazzo JP, Rosenberg A, Musiani P, Volinia S, Nenci I, Calin GA, Querzoli P, Negrini M, Croce CM: MicroRNA gene expression deregulation in human breast cancer. Cancer Res 2005;65:7065-7070.

-5 Calin GA, Dumitru CD, Shimizu M, Bichi R, Zupo S, Noch E, Aldler H, Rattan S, Keating M, Rai K, Rassenti L, Kipps T, Negrini M, Bullrich F, Croce CM: Frequent deletions and down-regulation of micro-RNA genes miR15 and miR16 at 13q14 in chronic lymphocytic leukemia. Proc Natl Acad Sci USA 2002;99:1552415529.

6 Guo J, Miao Y, Xiao B, Huan R, Jiang Z, Meng D, Wang Y: Differential expression of microRNA species in human gastric cancer versus non-tumorous tissues. J Gastroen Hepatol 2009;24:652-657

7 Michael MZ, O`Connor SM, van Holst Pellekaan NG, Young GP, James RJ: Reduced Accumulation of Specific MicroRNAs in Colorectal Neoplasia. Mol Cancer Res 2003;1:882-891.

-8 Yoon SO, Chun SM, Han EH, Choi J, Jang SJ, Koh SA, Hwang S, Yu E: Deregulated expression of microRNA-221 with the potential for prognostic biomarkers in surgically resected hepatocellular carcinoma. Hum Pathol 2011;42:1391-1400.

\9 Zhou B, Li C, Qi W, Zhang Y, Zhang F, Wu JX, Hu YN, Wu DM, Liu Y, Yan TT, Jing Q Liu MF, Zhai QW: Downregulation of miR-181a upregulates sirtuin-1 (SIRT1) and improves hepatic insulin sensitivity. Diabetologia 2012;55:2032-2043.

10 Bai H, Cao Z, Deng C, Zhou L, Wang C: miR-181a sensitizes resistant leukaemia HL-60/Ara-C cells to Ara-C by inducing apoptosis. J Cancer Res Clin 2012;138:595-602.

11 Li H, Hui L, Xu W: miR-181a sensitizes a multidrug-resistant leukemia cell line K562/A02 to daunorubicin by targeting BCL-2. Acta Bioch Bioph Sin 2012;44:269-277. 
12 Zhu DX, Zhu W, Fang C, Fan L, Zou ZJ, Wang YH, Liu P, Hong M, Miao KR, Liu P, Xu W, Li JY: miR-181a/b significantly enhances drug sensitivity in chronic lymphocytic leukemia cells via targeting multiple antiapoptosis genes. Carcinogenesis 2012;33:1294-1301.

-13 Zhuo L, Liu J, Wang B, Gao M, Huang A: Differential miRNA expression profiles in hepatocellular carcinoma cells and drug-resistant sublines. Oncol Rep 2013;29:555-562.

14 Lu Y, Roy S, Nuovo G, Ramaswamy B, Miller T, Shapiro C, Jacob ST, Majumder S: Anti-microRNA-222 (antimiR-222) and-181B suppress growth of tamoxifen-resistant xenografts in mouse by targeting TIMP3 protein and modulating mitogenic signal. J Biol Chem 2011;286:42292-42302.

15 Yu B, Sun X, Shen HY, Gao F, Fan YM, Sun ZJ: Expression of the apoptosis-related genes BCL-2 and BAD in human breast carcinoma and their associated relationship with chemosensitivity. J Exp Clin Cancer Res 2010;29:1-7.

16 Zhu W, Shan X, Wang T, Shu Y, Liu P: miR-181b modulates multidrug resistance by targeting BCL2 in human cancer cell lines. Int J Cancer 2010;127:2520-2529.

17 Chen G, Zhu W, Shi D, Lv L, Zhang C, Liu P, Hu W: MicroRNA-181a sensitizes human malignant glioma U87MG cells to radiation by targeting Bcl-2. Oncol Rep 2010;23:997-1003.

18 Shi L, Cheng Z, Zhang J, Li R, Zhao P, Fu Z, You Y: hsa-mir-181a and hsa-mir-181b function as tumor suppressors in human glioma cells. Brain Res 2008;1236:185-193.

19 Calin G A, Croce C M. MicroRNA signatures in human cancers. Nat Rev Cancer 2006;6:857-866.

20 Lashine YA, Seoudi AM, Salah S, Abdelaziz AI: Expression signature of microRNA-181-a reveals its crucial role in the pathogenesis of paediatric systemic lupus erythematosus. Clin Exp Rheumatol 2011;29:351357.

21 Ryan DG, Oliveira-Fernandes M, Lavker RM: MicroRNAs of the mammalian eye display distinct and overlapping tissue specificity. Mol Vis 2006;12:1175-1184.

22 Chen CZ, Li L, Lodish HF, Bartel DP: MicroRNAs modulate hematopoietic lineage differentiation. Science 2004;303:83-86.

23 Shin KH, Bae SD, Hong HS, Kim RH, Kang MK, Park NH: miR-181a shows tumor suppressive effect against oral squamous cell carcinoma cells by downregulating K-ras. Biochem Biophys Res Commun 2011;404:896-902.

24 Gao W, Shen H, Liu L, Xu J, Xu J, Shu Y: MiR-21 overexpression in human primary squamous cell lung carcinoma is associated with poor patient prognosis. J Cancer Res Clin 2011;137:557-566.

25 Ciafrè SA, Galardi S, Mangiola A, Ferracin M, Liu CG, Sabatino G, Negrini M, Maira G, Croce CM, Farace MG: Extensive modulation of a set of microRNAs in primary glioblastoma. Biochem Biophys Res Commun 2005;334:1351-1358.

26 Gao W, Yu Y, Cao H, Shen H, Li X, Pan S, Shu Y: Deregulated expression of miR-21, miR-143 and miR-181a in non small cell lung cancer is related to clinicopathologic characteristics or patient prognosis. Biomed Pharmacother 2010;64:399-408.

27 Marton S, Garcia MR, Robello C, Persson H, Trajtenberg F, Pritsch O, Rovira C, Naya H, Dighiero G, Cayota A: Small RNAs analysis in CLL reveals a deregulation of miRNA expression and novel miRNA candidates of putative relevance in CLL pathogenesis. Leukemia 2007;22:330-338.

28 Wang Y, Yu Y, Tsuyada A, Ren X, Wu X, Stubblefield K, Rankin-Gee EK, Wang SE: Transforming growth factor- $\beta$ regulates the sphere-initiating stem cell-like feature in breast cancer through miRNA-181 and ATM[J]. Oncogene 2010;30:1470-1480.

29 Lim LP, Lau NC, Garrett-Engele P, Grimson A, Schelter JM, Castle J, Bartel DP, Linsley PS, Johnson JM: Microarray analysis shows that some microRNAs downregulate large numbers of target mRNAs. Nature 2005;433:769-773.

-30 Elliott MJ, Murphy KM, Stribinskiene L, Ranganathan V, Sturges E, Farnsworth ML, Lock RB: Bcl-2 inhibits early apoptotic events and reveals post-mitotic multinucleation without affecting cell cycle arrest in human epithelial tumor cells exposed to etoposide. Cancer Chemoth Pharm 1999;44:1-11.

-31 Del Bufalo D, Biroccio A, Trisciuoglio D, Bruno T, Floridi A, Aquino A, Zupi G: Bcl-2 has differing effects on the sensitivity of breast cancer cells depending on the antineoplastic drug used. Eur J Cancer 2002;38:2455-2462.

-32 Yang CC, Hung PS, Wang PW, Liu CJ, Chu TH, Cheng HW, Lin SC: miR-181 as a putative biomarker for lymphnode metastasis of oral squamous cell carcinoma. J Oral Pathol Med 2011;40:397-404. 